\section{Cellular mechanisms of ALS mutations - a loss or a gain of function?}

\author{
C. Haass ${ }^{1,2}$ \\ 1 Adolf-Butenandt-Institute, Biochemistry, \\ Ludwig-Maximilians-University Munich \\ ${ }^{2}$ German Center for Neurodegenerative Diseases (DZNE), \\ Munich
}

TDP-43 and FUS are genetically and pathologically associated with amyotrophic lateral sclerosis (ALS) and frontotemporal lobar degeneration (FTLD). I will summarize our recent advances in understanding the cellular mechanisms of disease causing mutations in FUS and TDP-43 and show evidence, which may suggest a loss of function component in ALS/FTLD. A loss of function appears to be the case for the FUS associated ALS causing mutations. Consistent evidence from several laboratories demonstrates that these mutations reduce nuclear transport by disturbing a PY-nuclear localization signal, which leads to a cytoplasmic accumulation of the mutant proteins. Additional stressors are then required to initiate aggregation probably via stress granules.

Much less is known about the mechanism of TDP-43 mutations. We have recently generated zebrafish TDP-43 loss of function mutants. Homozygous loss of function mutations in zebrafish tardbp show no morphological phenotype due to compensation by a splice variant of tardbpl (Tar DNA binding protein of $43 \mathrm{kDa}$ like), a second zebrafish orthologue of human TARDBP. tardbp and tardbpl double homozygous mutants show muscle degeneration, strongly reduced blood circulation and a dramatic mispatterning of intersomitic vessels, impaired spinal motor axon outgrowth, and early death. A quantitative proteomic approach identified a muscle specific protein to be upregulated in tardbp and tardbpl double homozygous mutants. Strikingly, the same protein is similarly increased in the frontal cortex of FTLD-TDP patients suggesting aberrant expression in vascular smooth muscle cells. Thus, these findings reveal an unexpected role of TDP-43 in vascular patterning and muscle maintenance. Evidence will be presented that TDP-43 mutations only partially rescue the vascular phenotype.

Conflict of Interest: The author declares no conflict of interest.

\footnotetext{
Bibliography

DOI http://dx.doi.org/10.1055/s-0033-1346714

Drug Res 2013; 63, Suppl. 1: S17-S17

(c) Georg Thieme Verlag KG Stuttgart · New York .

ISSN 2194-9379
} 